\title{
Access to Stellar Population Models in the Virtual Observatory
}

\author{
Igor V. Chilingarian ${ }^{1,2,3}$ \\ ${ }^{1}$ Sternberg Astronomical Institute, Moscow State University, 13 Universitetsky prospect, \\ Moscow, 119992, Russia; email: chil@sai.msu.su \\ ${ }^{2}$ Centre de Recherche Astronomique de Lyon, Observatoire de Lyon, 9 Av. Charles André, \\ Saint-Genis Laval, F-69561, France; CNRS, UMR 5574; \\ ${ }^{3}$ Observatoire de Paris, LERMA, 61 Ave. de l'Observatoire, Paris, 75014, France
}

\begin{abstract}
A great effort is being made by the international Virtual Observatory community to build tools ready to be used by scientists. Presently, providing access to theoretical spectra in general, and synthetic spectra of galaxies in particular, is a matter of current interest in the Virtual Observatory. Several ways of accessing such spectra are available. We present two of them for accessing PEGASE.HR evolutionary synthesis models: HTTP-access to a limited number of parameters using Simple Spectral Access Protocol (SSAP), and full-featured WEB-service based access using Common Execution Architecture (CEA).
\end{abstract}

Keywords. astronomical data bases: miscellaneous, techniques: spectroscopic

\section{Introduction}

The main mission of the Virtual Observatory is to increase efficiency of scientific usage of astronomical data. International Virtual Observatory Alliance (IVOA $\dagger$ ) is a large international collaboration of national VO projects. Its main responsibility is to develop standards within IVOA working groups for the interoperability of archives, tools, services, and software, and to propose them to the corresponding division of IAU for further approval and recommendation.

Presently, the Virtual Observatory comprises:

- Archives and collections of science-ready astronomical data

- Data access services and analysis tools

- Resource registries to discover them

- Client software (interactive tools as well as client API)

- Set of standards used by all these resources to achieve interoperability

Establishing standards for accessing theory data is one of the cornerstones of present VO development.

\section{Access methods}

Here we present two possible approaches of accessing SSP models. The PEGASE.HR package (Le Borgne et al. 2004) is taken as an example. Both methods of accessing PEGASE.HR are implemented in a frame of the VO Paris project (Simon et al. 2006) by the author.

TSAP Access: a subset of the IVOA Simple Spectral Access Protocol (SSAP, Tody et al. 2006). Parameters are passed in the HTTP-GET query. Interface is easy to implement. Interactive clients, such as ESA VOSpec (http://esavo.esa.int/vospec/) are able to

\section{$\dagger$ http://www.ivoa.net/}


TSAP Access to PEGASE.HR http://vo.obspm.fr/cgi-bin/siap/pegasehr.pl

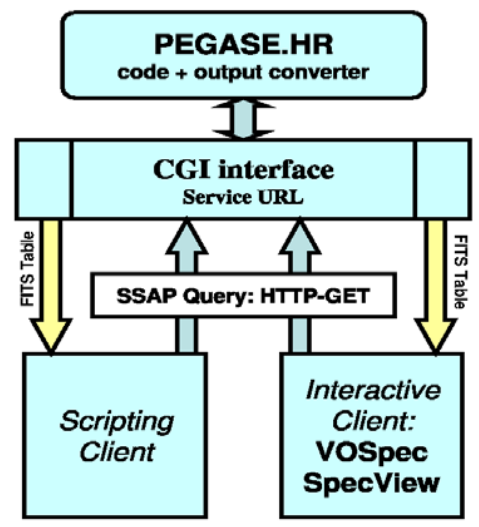

(a)
CEA Access to PEGASE.HR

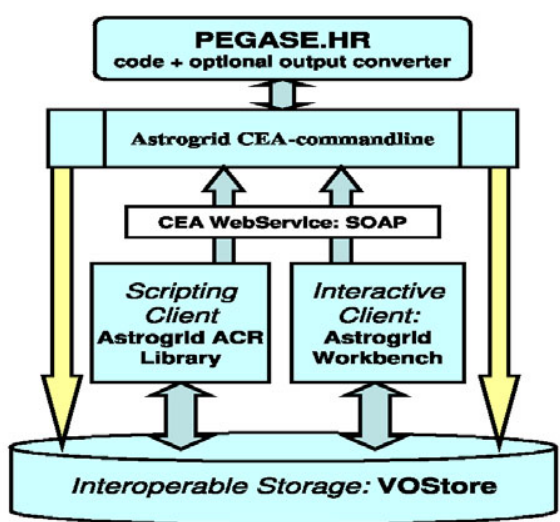

(b)

Figure 1. Schematic view of two ways of accessing PEGASE.HR simple stellar population models, available through the VO Paris portal.

build user interface dynamically, based on the response of the server providing capabilities (e.g. allowed query parameters). Direct download links to the FITS files, containing spectral models are provided. Scripting access is available using standard libraries dealing with HTTP protocol.

CEA Application Access: IVOA Common Execution Architecture is a standard way of accessing generic "legacy" applications and codes having CGI or command-line interfaces. CEA is based on the WebService access interface, thus parameters of the models and possible additional data are passed as a part of SOAP message, and no limitation on a number of parameters exists. Output is saved to the interoperable online storage: VOStore. Interactive client, ASTROGRID Workbench is available as well as the client library, ASTROGRID ACR Library, providing scripting level access from various programming languages, such as Java, $\mathrm{C} / \mathrm{C}++$, Perl, Python. Easy integration into workflows is a valuable advantage of this approach.

\section{Conclusion}

CEA Access is more versatile than TSAP, but more expensive in terms of infrastructure. It is preferable in case of complex models and if used in a workflow construction.

\section{Acknowledgements}

I am very grateful to the financial support, provided by the IAU for attending this symposium. Special thanks to Florence Durret (Institut d'Astrophysique de Paris) for additional funding.

\section{References}

Le Borgne, D. et al. 2004, A\&A 425, 881

Simon, G. et al. 2006, Proceedings of ADASS-XV, ASP Conference Series v. 351, 394

Tody, D. et al. 2006, SSAP v0.97, IVOA Working Draft 